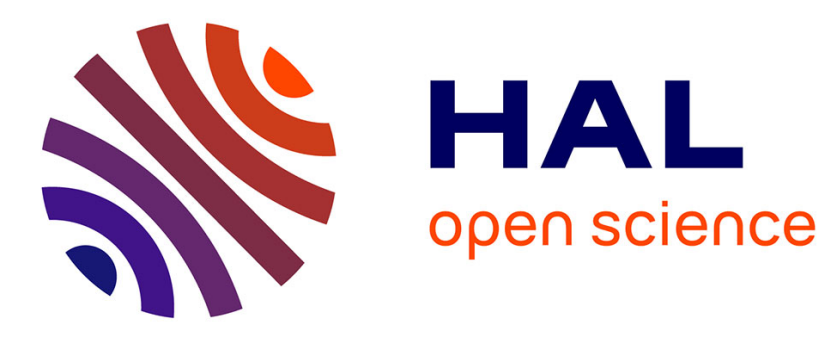

\title{
Les processus d'attribution de punitions. Étude des relations entre gravité de l'infraction pénale, caractéristiques de l'auteur, émotions et motivations à punir
}

Zlatka Atanasova-Denié, Manuel Tostain

\section{To cite this version:}

Zlatka Atanasova-Denié, Manuel Tostain. Les processus d'attribution de punitions. Étude des relations entre gravité de l'infraction pénale, caractéristiques de l'auteur, émotions et motivations à punir. Les cahiers Internationaux de Psychologie Sociale, 2008, 2 (78), pp.21-34. 10.3917/cips.078.0021. hal-01668280

\section{HAL Id: hal-01668280 \\ https://hal.science/hal-01668280}

Submitted on 19 Dec 2017

HAL is a multi-disciplinary open access archive for the deposit and dissemination of scientific research documents, whether they are published or not. The documents may come from teaching and research institutions in France or abroad, or from public or private research centers.
L'archive ouverte pluridisciplinaire HAL, est destinée au dépôt et à la diffusion de documents scientifiques de niveau recherche, publiés ou non, émanant des établissements d'enseignement et de recherche français ou étrangers, des laboratoires publics ou privés. 


\title{
Les processus d'attribution de punitions Étude des relations entre gravité de l'infraction pénale, caractéristiques de l'auteur, émotions et motivations à punir
}

\author{
Zlatka ATANASOVA-DENIÉ et Manuel TOSTAIN \\ Université de Caen Basse-Normandie, Caen, France
}

\begin{abstract}
Résumé : une recherche a été entreprise afin de préciser les relations entre differents déterminants, cognitifs, affectifs et motivationnels, de la punition. Dans ce cadre, nous avons présenté à des étudiants de 1ère année de Sciences Humaines des infractions pénales dans lesquelles on manipulait la gravité des conséquences pour la victime et les caractéristiques de l'auteur de l'infraction (personne avec ou sans troubles psychiatriques, primo-délinquant ou récidiviste). Ensuite, les sujets devaient répondre à des questions portant sur la responsabilité de l'auteur, les sentiments éprouvés à l'égard de l'auteur et de la victime, la punition à attribuer et différentes motivations à punir (rétributive, compensative, dissuasive et réhabilitative). Les résultats, qui vont dans le sens des hypothèses, mettent en évidence : 1) des liens entre facteurs cognitifs et affectifs ; 2) que les motivations à punir influencent l'attribution de punition; 3) que l'activation de chacune des motivations à punir résulte de facteurs cognitifs et affectifs en partie différents.
\end{abstract}

Mots-clés : attribution de punitions, facteurs cognitifs et affectifs, motivations à punir.

Régulièrement, nous sommes informés par les médias de délits et de crimes plus ou moins graves qui suscitent en nous interrogations, émotions et désir de punition. Face à ces actes qui froissent la conscience commune et qui constituent des transgressions des normes sociales, on peut ressentir un sentiment d'injustice et de crainte : des personnes sont injustement volées, blessées voire tuées et nous nous représentons le risque que nous-mêmes ou des êtres chers soient également victimes de tels agissements. Nous cherchons à comprendre pourquoi ces actes ont été commis, s'ils auraient pu être évités. On veut savoir si l'auteur était ou non en pleine possession de ses moyens et doit en conséquence répondre ou non de son acte. En cas d'acte particulièrement grave, nous pouvons éprouver de la colère s'il s'avère que l'auteur peut être tenu pour pleinement responsable de ce qu'il a fait et nous voulons que justice soit faite, ce qui passe le plus souvent par le désir de punir l'auteur d'un tel acte. Ce désir de punir peut être motivé par le fait que nous voulons faire payer l'auteur pour ce qu'il a fait ou encore pour empêcher que de tels actes ne se reproduisent.
Ainsi, différents éléments, d'ordres sociaux, cognitifs, affectifs et motivationnels, peuvent concourir à la punition (Fischer, 2003). D'un point de vue psychologique, il convient cependant de s'interroger plus précisément sur ce qui détermine et justifie la punition. Dans ce cadre, l'objectif de cet article est d'étudier chez les individus tout-venant les processus qui conduisent à l'attribution d'une punition et d'analyser les relations entre facteurs cognitifs, affectifs et motivationnels pouvant être impliqués dans cette attribution de punition. Plus précisément, nous chercherons à montrer que l'attribution par les individus d'une punition peut, pour partie, être envisagée comme la résultante d'une séquence temporelle et causale ordonnée en étapes (chaque étape agissant sur la suivante) dans laquelle interviennent successivement la gravité de l'infraction pénale, les caractéristiques de l'auteur de l'infraction pénale, les émotions éprouvées à l'égard de l'auteur de l'infraction et de la victime et les motivations à punir des individus. À cette occasion, nous souhaiterions développer l'idée que les motivations à punir varient selon les caractéristiques relatives de l'infraction. Pour terminer, nous souhaiterions que ce travail soit l'occasion de montrer l'intérêt, pour mieux comprendre les processus d'attribution de punitions, d'articuler deux champs d'études pour lesquels les points de contacts sont encore assez rares, le premier qui concerne l'attribution de responsabilité et de punition, le second qui porte sur les motivations à punir.

\section{Les recherches sur l'attribution de responsabilité et de punition}

À la suite de Piaget (1932) et de Heider (1944, 1958), de nombreuses recherches sur les processus d'attribution de responsabilité et de punition ont été réalisées. Ces travaux s'inscrivent principalement dans le champ de l'attribution causale qui étudie la façon dont les individus infèrent les causes des événements et des comportements et les effets de ces

Pour toute correspondance relative à cet article, s'adresser à Manuel Tostain, Université de Caen, Campus 1, U.F.R. de Psychologie, Bureau SE 61 1, Esplanade de la Paix, 14032 Caen CEDEx, France ou par courriel à <manuel.tostain@unicaen.fr>. 
inférences causales sur l'action. Ces travaux montrent que généralement l'attribution d'une punition est fonction d'une analyse préalable du niveau de responsabilité de l'auteur de l'infraction. Pour établir ce niveau de responsabilité (i.e. légitimité d'avoir à répondre de ses actes et à supporter une punition) (Viney, 1990), les individus s'appuient en premier lieu sur une recherche d'informations à propos des caractéristiques légales de l'infraction (c'est-à-dire reconnus comme valides dans un cadre judiciaire) : ainsi, on estime le degré d'implication causale de l'auteur dans la commission de l'infraction pénale (Shultz, Schleifer et Altman, 1981), le caractère intentionnel de l'acte (capacité à contrôler ses actes, à anticiper les conséquences de ses actes, caractère volontaire de l'acte) (Alicke, 1990), le statut ou non de récidiviste de l'auteur (Finkel, Maloney, Valbuena et Groscup, 1996). Les individus prennent également en compte l'importance du mal commis (Robbennolt, 2000). En accord avec le sens commun et les dispositions légales, ces travaux confirment que plus l'infraction est grave et l'auteur jugé responsable, et plus les peines sont sévères. Les individus prennent cependant parfois aussi en considération des facteurs extra-légaux (non valides ou problématiques dans un cadre judiciaire) tels que les appartenances sexuée (Steffensmeier et Kramer, 1982) et sociale de l'auteur (Hamilton et Sanders, 1981). Dans ce champ d'étude différents modèles d'attribution causale, qui postulent des processus par étapes, ont été développés (Fincham et Jaspars, 1980 ; Shaver, 1985; Weiner, 1995), ces différents modèles ayant pour point commun de mettre en relation niveau de responsabilité et sévérité de la punition. Par exemple, si on se réfère à Shaver (1985), quand on doit juger quelqu'un, une fois qu'on a pris connaissance de la gravité de l'infraction, on vérifie d'abord l'existence d'un lien causal entre l'auteur et les faits reprochés. Ensuite, si cette condition est remplie, on évalue le degré de contrôle que l'auteur avait sur ses actes et son intentionnalité (la conséquence de l'infraction était-elle voulue) afin d'établir son niveau de responsabilité. Ce niveau de responsabilité sera ensuite modulé par la présence éventuelle de circonstances atténuantes. Enfin, un blâme plus ou moins important est attribué en fonction de ce niveau de responsabilité. Certaines recherches interrogent cependant l'approche cognitive de ces modèles. D'abord, Robbennolt (2000) relève, contrairement à ce qu'indiquent ces modèles, qu'il n'y a pas toujours de liens entre le niveau de responsabilité et la sévérité de la punition. Ainsi, on a constaté que la punition, à intentionnalité constante, est souvent d'autant plus sévère que les dommages causés sont importants (Mazzocco, Alicke et Davis, 2004). En particulier, on attribue une punition plus sévère pour des dommages importants, même si ces dommages sont d'origine accidentelle (ne résultant pas de la volonté des auteurs des actes incriminés). C'est ce que l'on appelle le biais lié aux conséquences (en anglais « outcome bias »). Ensuite, les étapes postulées par les modèles d'attribution causale ne sont pas toujours respectées. Ainsi, Oswald (Oswald, Orth, Aeberhard et Schneider, 2005) met en évidence, en manipulant la gravité de l'infraction, que l'importance des conséquences de l'infraction (pour la victime) peut agir directement sur la sévérité de la punition sans nécessairement transiter par la responsabilité. Pour expliquer ce résultat, cette auteure évoque le fait que la sévérité de la punition n'est pas uniquement fonction de l'analyse préalable de la responsabilité mais peut être motivée par d'autres facteurs comme la prise en compte de la souffrance des victimes ou la volonté de faire un exemple. Oswald soulève ici la question du rôle des motivations à punir sur la punition.

On s'est également intéressé aux aspects affectifs de la responsabilité. Ici, il a été montré que l'attribution de responsabilité génère certains états émotifs chez les observateurs. Par exemple, Alicke (2000) rappelle le fait que les auteurs de crimes suscitent d'autant plus de colère qu'ils sont estimés responsables de ce qu'ils ont fait. D'autre part, les émotions peuvent avoir une influence importante sur le désir de punir. Là, ces recherches s'inspirent du travail classique de Fauconnet (1920). Pour cet auteur, les infractions (en particulier les crimes) qui constituent une transgression des normes sociales, violent des états forts de la conscience collective et rompt l'équilibre de son ordonnancement; d'où la nécessité, ensuite, de liquider les conséquences émotionnelles ainsi produites. L'émotion soulevée est alors apaisée en se dépensant par le moyen de la punition. Dans ce cadre, on peut citer les travaux de Haidt, Koller et Dias (1993) qui montrent que les actes qui outragent la morale suscitent des affects fortement négatifs (de dégout, de rage). Ces affects négatifs sont à l'origine d'un désir de punition qui a pour but d'annuler symboliquement l'offense produite. Tetlock (Golberg, Lerner et Tetlock, 1999; Lerner, Golberg et Tetlock, 1998), dans une optique assez proche de celle de Lerner (1998), met en évidence que plus l'infraction pénale est associée à une injustice élevée (l'auteur restant par exemple impuni), plus cela suscite de la colère chez l'observateur et plus celui-ci est amené à attribuer des peines sévères face à des infractions pénales ultérieures. Cette orientation punitive traduit le désir de rendre 
le mal pour le mal, de faire payer l'auteur pour le mal commis, ce qui pose la question des motivations à punir, point que nous allons maintenant aborder.

\section{Les recherches sur les motivations à punir}

Si de nombreux travaux ont été réalisés sur les processus d'attribution de responsabilité et de punition, on s'est moins interrogé sur les raisons, les motivations des individus à punir et sur les objectifs que la punition doit remplir d'après les individus (pourquoi faut-il punir? Qu'est-ce qui légitime que l'on punisse ? Dans quel but ?) (Van de Kerchove, 2005). On s'est également peu interrogé sur les effets des motivations à punir sur la sévérité de la punition (Vidmar et Miller, 1980). Afin de préciser les motivations à punir des individus, il peut être utile au préalable d'examiner comment les philosophes et les théoriciens de la justice pénale justifient la punition (Larguier, 2001).

Classiquement, si on se réfère aux travaux de philosophie pénale (Garapon, Gros et Pech, 2001 ; Villey, 2003), on distingue principalement quatre justifications théoriques de la peine ou philosophies pénales. La première philosophie, dite rétributive (qualifiée de Fust Deserts Theory par les auteurs anglo-saxons), pose que l'individu doit répondre de ses actes car il est un être de raison, libre de commettre ou non tel ou tel acte. Dans cette philosophie, la punition sert à punir le mal commis et sera d'autant plus forte que l'auteur sera jugé responsable, c'est-à-dire aura agi sans contraintes (volontairement) et que le mal qu'il a causé est important (Hegel, 1821 ; Kant, 1796). À côté de cette philosophie à caractère rétrospectif (on se réfère à ce qui est situé en amont de l'acte), il y a une seconde philosophie, au contraire prospective (se projetant en aval de l'acte) dite dissuasive ou préventive (en anglais Deterrence Theory). Ici, la punition sert à défendre la société, à assurer l'harmonie sociale et à prévenir le crime (Beccaria, 1764 ; Bentham, 1789). Selon cette philosophie, punir a pour objectif de dissuader l'auteur de recommencer (en anglais specific deterrence) ou de décourager d'autres individus de commettre des actes similaires (en anglais general deterrence). Pour cette philosophie, on peut dire qu'une punition juste, c'est une punition suffisamment forte pour être dissuasive. La troisième philosophie, dite réhabilitative, met en avant la vertu rééducative et resocialisatrice de la punition (Saleilles, 1898 ; Tocqueville, 1833). La peine a pour fonction d'inciter l'individu qui, en commettant un crime, s'est exclu de la société, à réfléchir sur son acte, à changer pour se réintégrer. Dans ce cadre, une punition juste, c'est une punition mesurée qui ne doit pas être trop forte (dans ce cas l'indi- vidu pourrait être découragé de changer). Enfin, la quatrième philosophie correspond à la philosophie compensative (Garapon et Salas, 1996). Cette philosophie se centre sur les victimes et repose sur un sentiment d'identification et de sympathie à l'égard des victimes. Dans ce cadre, la punition doit permettre aux victimes d'être reconnues en tant que victimes par la société et de dépasser leurs souffrances (ou celles de leurs proches). Ici, une punition juste, c'est une punition proportionnelle à leurs souffrances, le malheur qu'apporte la punition à l'auteur de l'acte criminel ayant pour objectif d'essayer de compenser le malheur des victimes. Cette dernière philosophie est parfois assez proche d'une logique vindicative et elle se traduit par un désir de punition souvent très fort (Barillon et Bensussan, 2004). Des travaux montrent que cette philosophie prend une place de plus en plus importante au niveau social et pénal en raison de l'attention actuelle aux victimes (Cesoni et Rechtman, 2005 ; Rauschenbach et Languin, 2007 ; Salas, 2005). Si on essaie de résumer ces quatre philosophies, on peut dire qu'elles renvoient à deux grands types de philosophies pénales, un type rétributif au sens large centré sur le mal commis (rétribution de l'auteur et compensation de la victime), un type utilitariste centré sur les moyens d'assurer l'harmonie sociale (dissuasion et réhabilitation).

Différents travaux, la plupart récents, montrent que les philosophies pénales à la fois se retrouvent dans les réflexions des individus ordinaires et constituent une systématisation théorique des motivations à punir des individus (Carlsmith, Darley et Robinson, 2002 ; De Keïser, Van der Leeden et Jackson, 2002 ; Oswald, Hupfeld, Klug et Gabriel, 2002).

En psychologie, on doit à Hogarth (1971) d'avoir été un des premiers à s'intéresser à l'influence des motivations à punir sur l'attribution de punition. Son étude, qui reposait sur l'interview de magistrats canadiens, montrera que plus les juges insistaient sur la motivation réhabilitative et plus ils prononçaient des punitions faibles. À l'inverse, plus les juges mettaient en avant des motivations rétributives et dissuasives et plus ils proposaient des punitions élevées. McFatter (1978), dans un cadre expérimental, reproduira ces résultats et montrera notamment que la motivation réhabilitative est associée à une minimisation de la responsabilité de l'auteur. Depuis ces deux recherches princeps, les travaux de psychologie sociale ont été peu nombreux. On peut citer le travail de Darley, Carlsmith et Robinson (2000) qui montre que plus l'infraction constitue un outrage au principe moral de respect de l'intégrité d'autrui et 
plus la motivation rétributive est mobilisée et plus la sévérité de la punition augmente. En ce qui concerne la motivation dissuasive, on a mis en évidence que plus les crimes sont fréquents, plus l'individu mis en cause est connu pour avoir commis déjà des actes similaires, et plus on met l'accent sur cette motivation pour justifier la punition (Weiner, 2001). Toujours à propos de cette motivation dissuasive, les recherches montrent que l'adhésion à cette motivation a pour effet de recommander des punitions sévères car seules jugées efficaces pour empêcher les auteurs de recommencer (Rucker, Polifroni, Tetlock et Scott, 2004 ; Tyler et Boeckmann, 1997).

Les travaux issus du champ de l'attribution de responsabilité et de punition et du champ des motivations à punir mettent en évidence toute une série de facteurs impliqués dans la détermination de punition. D'un côté, le premier champ d'études souligne le rôle joué par la gravité de l'infraction et par les facteurs d'ordre cognitif (analyse de la responsabilité) et affectif (impact des émotions). D'un autre côté, le second champ d'études montre l'importance de différentes motivations à punir sur la punition. Pour autant, en raison de l'absence de points de contacts entre ces deux champs d'études, les travaux n'analysent qu'une partie des déterminants de la punition. Il faut cependant citer le travail de Weiner qui fait figure d'exception (Graham, Weiner et Zucker, 1997). Cet auteur propose en effet un modèle d'attribution de punition qui articule les facteurs cognitifs, affectifs et motivationnels en s'appuyant sur les analyses issues des deux champs d'études que l'on vient de mentionner. Dans ce modèle, l'attribution de la punition ferait suite à une série d'étapes qui impliquent successivement: (1) l'évaluation de la responsabilité de l'auteur de l'infraction et de son passé judiciaire, (2) les émotions à l'égard de l'auteur, (3) les motivations à punir de la personne qui juge. Plus précisément, quand un individu, un observateur a à juger une infraction et à attribuer une punition, il mettrait en œuvre deux processus parallèles. Le premier processus aurait pour point de départ, comme l'indique les théories de l'attribution causale, la recherche d'informations relatives à la responsabilité de l'auteur de l'infraction. Dans ce cadre, l'observateur commencerait par déterminer la contrôlabilité de l'auteur qui renvoie à l'intégrité des facultés intellectuelles, à la capacité de décider librement et volontairement de ses actes. On parlera de contrôlabilité forte quand l'auteur était en pleine possession de ses moyens intellectuels au moment des faits, de contrôlabilité faible quand les facultés intellectuelles de l'auteur étaient, au contraire, altérées. Ensuite, l'observateur, à partir de ce niveau de contrôlabilité estimé, déterminerait le niveau de responsabilité attribué à l'auteur, jugeant celui-ci d'autant plus responsable que son niveau de contrôlabilité estimé est fort. Ce niveau de responsabilité s'associerait ensuite à des émotions plus ou moins négatives. En l'occurrence, plus on jugera que l'auteur est responsable de ses actes, a volontairement transgressé la loi, et plus on éprouvera à son égard des sentiments de colère : l'injustice consécutive à l'acte soulève d'autant plus d'émotions qu'elle a été voulue. Puis, ces sentiments négatifs détermineraient l'activation de buts rétributifs : l'auteur doit payer pour ce qu'il a fait. Enfin, plus ces buts rétributifs seraient activés et plus la punition recommandée serait sévère. Le second processus reposerait sur l'analyse par l'observateur de la stabilité qui correspond au passé judiciaire de l'auteur. On parlera de stabilité faible quand c'est la première fois que l'auteur commet une infraction, de stabilité forte quand l'auteur a déjà commis par le passé de nombreuses infractions du même type. Ensuite, la stabilité servirait à estimer la probabilité de récidive de l'auteur : cette probabilité de récidive sera jugée d'autant plus forte que l'observateur estime élevée la stabilité du comportement infractionnel. Cette probabilité de récidive poserait pour l'observateur la question de la dimension dissuasive de la peine (punir pour éviter que l'auteur ne recommence). Enfin, cette focalisation sur la dimension dissuasive amènerait l'observateur à proposer des punitions sévères pour rompre avec le cercle de la récidive : les peines attribuées précédemment à l'auteur n'ont pas suffit, il faut donc qu'elles soient encore plus sévères. Les différentes expériences qui ont été mises en œuvre par cet auteur (Weiner, Graham et Reyna, 1997) confirment ce schéma causal impliquant deux processus, l'un axé sur la dimension rétributive, l'autre axé sur la dimension dissuasive.

\section{Objectif de la recherche}

Certains facteurs, qui nous semblent importants, ne sont cependant pas évoqués dans le modèle de Weiner. Ainsi, Weiner ne prend pas en compte la gravité des infractions. Or, comme le suggèrent différents travaux, la gravité peut avoir des effets directs sur la punition sans nécessairement transiter par la responsabilité (Oswald et al., 2005 ; Robbennolt, 2000). De plus, la prise en considération de la gravité permettrait de préciser l'influence d'une motivation généralement ignorée dans les recherches psychosociales, à savoir la motivation compensative. Il nous semble important de tenir compte de cette motivation dans la mesure où elle est de plus en plus 
présente au niveau social et pénal (Cesoni et Rechtman, 2005). Rappelons que cette motivation s'appuie sur une certaine identification à la victime et sur la sympathie éprouvée à son égard, et que la punition sert, comme l'indique le nom de cette motivation, à compenser la souffrance de la victime. Ajoutons que par définition, cette souffrance dépend ellemême de la gravité de l'infraction, de l'importance des conséquences pour la victime. Compte tenu de cela, il nous semble que l'on pourrait envisager, à côté des processus évoqués par Weiner, le schéma causal suivant : d'abord, on peut s'attendre à ce que plus le mal commis (en cas d'atteintes aux personnes) est jugé important par l'observateur et plus celui-ci aura tendance à considérer que la souffrance de la victime est élevée. Ensuite, on peut s'attendre, dans la logique de la motivation compensative, à ce que plus la souffrance de la victime est jugée élevée et plus la victime suscite des sentiments de sympathie chez l'observateur. En conséquence, on peut supposer que ces sentiments de sympathie incitent l'observateur à se focaliser sur la dimension compensative de la punition. Enfin, si on en croit les analyses sociologiques qui indiquent que l'orientation répressive actuelle s'explique pour partie par la focalisation contemporaine sur la victime (Salas, 2005), on peut s'attendre à ce que la punition soit d'autant plus élevée que cette motivation compensative est mobilisée. Par ailleurs, il conviendrait de faire la distinction entre motivation dissuasive individuelle (punir pour dissuader l'individu de recommencer) et motivation dissuasive collective (punir pour dissuader d'autres individus de commettre des actes similaires). Si ces deux motivations d'inspiration utilitariste s'inscrivent dans une logique répressive (Lagrange, 2003), les travaux actuels ne précisent généralement pas l'influence spécifique de ces deux motivations sur la sévérité de la punition. Enfin, on peut supposer qu'une autre motivation utilitariste, la motivation réhabilitative (punir pour inciter l'individu à faire retour sur son geste afin qu'il se réintègre ensuite dans la communauté) puisse avoir des effets sur la punition. Ici, rappelons que cette motivation repose sur l'idée humaniste que l'individu peut changer, qu'il faut lui donner une nouvelle chance, ce que l'on considère comme d'autant plus possible que sa faute est exceptionnelle, qu'il ne présente pas un parcours infractionnel persistant et répété (Saleilles, 1998). Par ailleurs, les travaux montrent que cette motivation s'associe à des peines moins sévères (Hogarth, 1971 ; McFatter, 1978). Dès lors, nous nous proposerons également de tester un schéma causal associé à la motivation réhabilitative. Dans la mesure où cette motivation est une motivation utilitariste, ce schéma reposera au départ sur les mêmes dimensions que celles évoquées par Weiner pour la motivation utilitariste dissuasive, à savoir la stabilité et la probabilité de récidive. Dans ce cadre, le schéma causal proposé serait le suivant : l'estimation de la stabilité du comportement de l'auteur de l'infraction déterminerait la probabilité de récidive (plus la stabilité sera vue comme faible et plus la probabilité de récidive sera jugée faible). Ensuite, compte tenu du fait que si l'auteur n'est pas engagé de façon répétée dans la délinquance, ce qui rend plus plausible le sentiment que les choses peuvent encore changer, que l'auteur peut se réintégrer, on postulera qu'une probabilité de récidive faible encouragera la prise en compte de la motivation réhabilitative. Enfin, compte tenu des travaux mettant en évidence le lien négatif entre réhabilitation et punition, on peut supposer que plus l'observateur aura recours à la motivation réhabilitative et plus la sévérité de la punition sera moindre.

Dans ce cadre, nous avons mis en œuvre une expérience dans laquelle on présentait un fait divers (un vol avec agression physique) qui faisait l'objet de trois manipulations expérimentales. Une manipulation de l'importance des conséquences pour la victime (faibles us fortes) afin de faire varier la perception de la gravité de l'acte. Une manipulation du statut mental de l'auteur (selon les versions l'auteur de l'acte était présenté comme en pleine possession de ses moyens ou comme une personne atteinte d'un traumatisme psychique) afin de faire varier la perception de la contrôlabilité de l'auteur. Enfin, une manipulation du passé judiciaire de l'auteur (primodélinquant vs récidiviste) afin de faire varier la perception de la stabilité du comportement infractionnel de l'auteur. Ensuite, les sujets devaient estimer la gravité de l'acte, la contrôlabilité et la stabilité du comportement infractionnel de l'auteur, la responsabilité de l'auteur, la souffrance de la victime, la probabilité de récidive de l'auteur, la colère éprouvée à l'égard de l'auteur, la sympathie à l'égard de la victime, la sévérité de la punition. Enfin, les sujets devaient indiquer leur degré d'accord avec cinq motivations à punir (rétributive, compensative, dissuasives individuelle et collective, réhabilitative).

\section{Hypothèses}

En référence au travail de Weiner, nous postulerons un processus d'attribution de punition résultant d'étapes ordonnées successives et différentes pour chaque motivation. Nous prendrons en compte les motivations rétributive, dissuasives individuelle 
et collective, réhabilitative et compensative. Au regard des travaux sur le biais lié aux conséquences (Robbennolt, 2000) et de la recherche d'Oswald (Oswald et al., 2005), une hypothèse impliquant un lien direct entre la gravité de l'infraction et la punition sera proposée (hypothèse 6).

- H1 : relative à la motivation rétributive : plus la contrôlabilité de l'auteur sera jugée forte et plus la responsabilité de l'auteur tendra à être élevée. Ce niveau de responsabilité déterminera la colère éprouvée à l'égard de l'auteur (d'autant plus forte que la responsabilité sera jugée élevée). Ensuite, plus la colère sera forte et plus l'observateur sera incité à punir pour des motifs rétributifs. Enfin, plus la motivation rétributive sera mobilisée (scores élevés) et plus la punition sera sévère.

- H2 : relative à la motivation compensative : plus la gravité de l'infraction sera jugée forte et plus la souffrance de la victime sera estimée importante. Plus cette souffrance sera estimée importante et plus le niveau de sympathie pour la victime sera élevé, ce qui entrainera une mobilisation d'autant plus forte de la motivation compensative. Enfin, plus la motivation compensative sera mobilisée et plus la punition sera sévère.

- H3 et $\mathbf{H 4}$ : relatives respectivement aux motivations dissuasives individuelle et collective. En raison de l'absence de données dans la littérature distinguant précisément ces deux motivations, les deux hypothèses seront identiques :

Plus le comportement de l'auteur de l'infraction sera jugée stable et plus la probabilité qu'il récidive sera considérée comme élevée. Ensuite, plus la probabilité de récidive sera élevée et plus les motivations dissuasives individuelle $(\mathbf{H 3})$ et collective $(\mathbf{H 4})$ seront mobilisées. Enfin, la punition sera d'autant plus sévère que ces motivations dissuasives seront mobilisées.

- H5 : relative à la motivation réhabilitative : moins le comportement de l'auteur de l'infraction sera jugée stable et moins la probabilité de récidive sera jugée élevée. Ensuite, moins la probabilité de récidive sera jugée élevée et plus la motivation réhabilitative sera mobilisée. Enfin, la punition sera d'autant moins sévère que la motivation réhabilitative sera mobilisée.

H6 : relative au biais lié aux conséquences (lien direct entre gravité et punition) : plus la gravité de l'infraction sera jugée forte et plus la sévérité de la punition sera élevée.
On peut résumer les différentes hypothèses par les schémas suivants :

$\mathrm{H} 1:$ contrôlabilité $\rightarrow$ responsabilité $\rightarrow$ colère auteur $\rightarrow$ rétribution $\rightarrow$ punition.

$\mathrm{H} 2$ : gravité $\rightarrow$ souffrance de la victime $\rightarrow$ sympathie victime $\rightarrow$ compensation $\rightarrow$ punition.

H3 : stabilité $\rightarrow$ probabilité récidive $\rightarrow$ dissuasion individuelle $\rightarrow$ punition.

H4 : stabilité $\rightarrow$ probabilité récidive $\rightarrow$ dissuasion collective $\rightarrow$ punition.

H5 : stabilité $\rightarrow$ probabilité récidive $\rightarrow$ réhabilitation $\rightarrow$ punition.

H6 : gravité $\rightarrow$ punition.

\section{Méthode}

Participants

402 étudiants en première année de psychologie (346 filles et 56 garçons ; âge moyen $=19,4$ ans ; $\sigma=1.6$ ans).

\section{Procédure et plan expérimental}

On présentait l'expérience comme une étude sur le jugement social. Les sujets étaient assignés aléatoirement à l'une des cellules d'un plan expérimental à 2 (importance des conséquences : faibles, fortes) x 2 (statut mental de l'auteur : personne en pleine possession de ses moyens, personne atteinte d'un traumatisme crânien) x 2 (passé judiciaire de l'auteur : primo-délinquant, récidiviste) facteurs inter-sujets. Les sujets commençaient par lire un scénario relatant ce qui était présenté comme un fait divers réel. Ce scénario évoquait un vol avec agression physique.

\section{Scénario et variables indépendantes}

«Sur le RER G (région parisienne), vers minuit, vendredi soir, dans une des voitures de la rame, une femme qui était en train de compter son argent a été agressée par un homme ».

\section{- Importance des conséquences}

Puis à la suite de la phrase « ... a été agressée par un homme », version 1 "...Celui-ci lui a arraché son sac des mains, puis s'est enfui à la station suivante » (conséquences faibles) ; version $2:$ «... Celui-ci, après l'avoir violemment frappée plusieurs fois au visage, lui a arraché son sac des mains, puis s'est enfui à la station suivante » (conséquences fortes) ${ }^{1}$.

Ensuite, le fait divers continuait par la description suivante : «...Un passager, situé dans la voiture d'à 
côté, a alerté avec son portable la police qui a pu arrêter l'homme. Une enquête préliminaire a été ouverte. Cette enquête met en évidence que l'homme et la femme étaient seuls dans la voiture au moment des faits ».

\section{- Statut mental de l'auteur}

À la suite de la phrase «... étaient seuls dans la voiture au moment des faits », version $1:$ «...Un examen médico-psychiatrique indique qu'il aurait agi en pleine possession de ses moyens (personne en pleine possession de ses moyens) ; version $2:$ «... Un examen médico-psychiatrique indique qu'il n'aurait pas agi en pleine possession de ses moyens. Quelques jours auparavant, suite à une chute, il aurait subi un traumatisme crânien l'amenant à ne plus agir normalement » (personne atteinte d'un traumatisme crânien).

\section{- Passé judiciaire de l'auteur}

Puis à la suite, version $1:$ «... l'enquête révèle également que l'homme n'a pas de casier judiciaire » (primo-délinquant) ; version $2:$ «... L'enquête révèle également que l'homme a déjà été arrêté à plusieurs reprises pour vols avec violence » (récidiviste).

\section{Variables dépendantes}

Ensuite, les sujets devaient estimer sur des échelles de type Likert en 7 points : (1) la gravité de l'infraction $(1=$ pas du tout grave $; 7=$ extrêmement grave); (2) la contrôlabilité de l'auteur ( $1=$ pas du tout le contrôle de ce qu'il faisait ; 7 = totalement le contrôle de ce qu'il faisait) ; (3) la stabilité du comportement infractionnel de l'auteur $(1=$ a l'habitude de respecter la loi ; 7 = n'a pas du tout l'habitude de respecter la loi); (4) la responsabilité de l'auteur (1 = pas du tout responsable ; $7=$ totalement responsable); (5) la souffrance de la victime ( $1=$ pas du tout de souffrance ; 7 = énormément de souffrance) ; (6) la probabilité de récidive de l'auteur $(1=$ pas du tout probable ; 7 = extrêmement probable) ; (7) la colère à l'égard de l'auteur ( 1 = pas du tout de colère ; 7 = énormément de colère); (8) la sympathie à l'égard de la victime ( 1 = pas du tout de sympathie ; 7 = énormément de sympathie); (9) la sévérité de la punition $(1=$ pas du tout sévère ; $7=$ extrêmement sévère) ; (10) le type de punition $(1=$ aucune punition ; $2=$ amende ; $3=3$ mois de prison avec sursis ; $4=3$ mois de prison ferme ; $5=6$ mois de prison ferme ; $6=1$ an de prison ferme ; $7=3$ ans de prison ferme). Pour terminer, les sujets devaient indiquer sur des échelles de type Likert (allant de 1 $=$ pas du tout d'accord à $7=$ tout à fait d'accord) leur degré d'accord avec les cinq motivations à pu- nir suivantes ${ }^{2}$ : (1) motivation rétributive : « Les individus doivent répondre de leurs actes. La punition que j'ai proposée a surtout pour raison d'être de faire payer l'auteur de l'acte pour ce qu'il a fait »; (2) motivation compensative : "La société doit rendre justice aux victimes. La punition que j'ai proposée a surtout pour raison d'être de compenser les torts subis par la victime »; (3) motivation dissuasive individuelle : "La société doit se protéger. La punition que j'ai proposée a surtout pour raison d'être de dissuader (par le risque d'une nouvelle peine) l'auteur de l'acte de recommencer »; (4) motivation dissuasive collective : «La société doit se protéger. La punition que j'ai proposée a surtout pour raison d'être de dissuader d'autres individus de faire la même chose "; (5) motivation réhabilitative : "La société ne doit pas exclure. La punition que j'ai proposée a surtout pour raison d'être d'aider l'auteur de l'acte à réfléchir pour qu'il change et puisse se réintégrer ensuite dans la société ». Afin de limiter un effet possible de l'ordre de passation des items de motivations à punir, les cinq items, en fonction des questionnaires, étaient présentés selon cinq ordres différents (ordre 12345, ordre 23451, ordre 34512, ordre 45123, ordre 51234).

Les traitements statistiques ne révèlent pas d'effets liés à l'ordre de présentation des items de motivations à punir ni d'effets liés au sexe des sujets. Cies deux variables n'ont donc pas été prises en compte dans la présentation des résultats.

\section{Résultats \\ Analyses préliminaires}

Pour chacune des variables dépendantes, nous avons procédé à une Anova 2 (importance des conséquences : faibles, fortes) x 2 (statut mental de l'auteur : personne en pleine possession de ses moyens, personne atteinte d'un traumatisme crânien) x 2 (passé judiciaire de l'auteur : primo-délinquant, récidiviste).

\section{Vérification de la manipulation des variables expérimentales}

Conformément à notre manipulation : 1) la perception de la gravité est plus élevée quand les histoires sont associées à des conséquences fortes $(M=4.85)$ qu'à des conséquences faibles $(M=3.50 ; F(1,394)$ $=123.27 ; p<.001)$; 2) la contrôlabilité est plus élevée quand l'auteur a agi en pleine possession de ses moyens $(M=6.43)$ que lorsqu'il aurait été victime d'un traumatisme crânien $(M=3.45 ; F(1,394)=$ $577.73 ; p<.001)$; 3) la stabilité est plus élevée dans le cas où l'auteur a déjà été arrêté, est récidiviste $(M$ 
$=5.10)$ que lorsqu'il n'a pas de casier judiciaire, est primo-délinquant $(M=2.45 ; F(1,394)=300.73$; $p<.001$ ) (voir tableau 1).

Jugements, émotions, motivations à punir et punition

\section{- Responsabilité de l'auteur}

L'auteur est jugé plus responsable, d'une part quand il a agi en pleine possession de ses moyens $(M=6.57)$ que lorsqu'il aurait été victime d'un traumatisme crânien $(M=4.20 ; F(1,384)=432.83 ; p<.001)$, d'autre part quand il est récidiviste $(M=5.57)$ que lorsqu'il est primo-délinquant $(M=5.20 ; F(1,394)$ $=7.21 ; p<.007)$.

\section{- Souffrance de la victime}

La souffrance de la victime est jugée plus importante en cas de conséquences fortes $(M=5.88)$ qu'en cas de conséquences faibles $(M=4.53 ; F(1,394)=$ $137.04 ; p<.001)$

\section{- Probabilité de la récidive}

La probabilité de la récidive est jugée plus forte pour le récidiviste $(M=5,93)$ que pour le primo-délinquant $(M=4.31 ; F(1,394)=196.65 ; p<.001)$.

\section{- Colère à l'égard de l'auteur}

La colère à l'égard de l'auteur est plus importante : 1) en cas de conséquences fortes $(M=4.76)$ qu'en cas de conséquences faibles $(M=4.02 ; F(1,394)=$ $23.31 ; p<.001) ; 2$ ) quand l'auteur a agi en pleine possession de ses moyens $(M=4.83)$ que lorsqu'il aurait été victime d'un traumatisme crânien $(M=$ $3.95 ; F(1,394)=34.53 ; p<.001)$.

\section{- Sympathie à l'égard de la victime}

La sympathie à l'égard de la victime est plus importante en cas de conséquences fortes $(M=5.09)$ qu'en cas de conséquences faibles $(M=4.63 ; F(1$, 392) $=11.309 ; p<.001)$.

\section{- Motivations à punir}

On remarque un effet de nos manipulations expérimentales sur la rétribution et la compensation. La note de rétribution est plus forte quand l'auteur était en pleine possession de ses moyens ( $M=5.25)$ que lorsqu'il aurait subi un traumatisme crânien $(M$ $=4.09 ; F(1,394)=50.41 ; p<.001)$. D'autre part, la note de compensation est plus élevée en cas de conséquences fortes $(M=4.20)$ que de conséquences faibles $(M=3.80 ; F(1,394)=5.88 ; p<.01)$. Par contre, en ce qui concerne les motivations utilitaristes (dissuasions individuelle, collective et réhabilita- tion), on ne constate pas d'effets significatifs de nos variables.

\section{- Sévérité de la punition}

On note un effet de chacune de nos trois variables sur la sévérité de la punition. Ainsi, la punition est plus sévère : 1) en cas de conséquences fortes $(M$ $=4.28)$ que de conséquences faibles $(M=3.54$; $F(1,394)=39.92 ; p<.001) ; 2)$ quand l'auteur a agi en pleine possession de ses moyens $(M=4.38)$ que lorsqu'il aurait subi un traumatisme crânien $(M=$ $3.43 ; F(1,394)=62.97 ; p<.001) ; 3)$ quand l'auteur est récidiviste $(M=4.34)$ que lorsqu'il est primo-délinquant $(M=3.47 ; F(1,394)=54.10 ; p<.001)$.

\section{- Type de punition}

On remarque également un effet de chacune de nos trois variables sur le type de punition. Le type de punition est plus important : 1) en cas de conséquences fortes $(M=5.14)$ qu'en cas de conséquences faibles $\left.\left(M=3.72^{3} ; F(1,394)=101.22 ; p<.001\right) ; 2\right)$ quand l'auteur a agi en pleine possession de ses moyens ( $M=4.76)$ que lorsqu'il aurait subi un traumatisme crânien $(M=4.08 ; F(1,394)=45.24 ; p<.001)$; 3) quand l'auteur est récidiviste $(M=4.79)$ que lorsqu'il est primo-délinquant $(M=4.05 ; F(1,394)$ $=37.68 ; p<.001)$.

\section{Analyse des pistes causales (" path analysis »)}

Afin de tester nos hypothèses et vérifier la présence éventuelle de liens non prévus, nous avons procédé à une analyse des pistes causales (Tabachnick et Fidell, 2001) en ajoutant au fur et à mesure de l'analyse des prédicteurs. Pour cela, nous avons ordonné l'analyse en fonction des différentes étapes envisagées par les hypothèses. Dans un premier temps, nous avons pris comme prédicteurs nos trois variables initiales (gravité, contrôlabilité et stabilité) et nous avons procédé à trois analyses de régression, une pour chacune des variables dépendantes suivantes : responsabilité de l'auteur, souffrance de la victime, probabilité de la récidive. Ensuite, nous avons ajouté ces trois variables dépendantes aux trois prédicteurs initiaux et nous avons effectué deux analyses de régression, une pour chacune des variables affectives : colère à l'égard de l'auteur, sympathie à l'égard de la victime. Puis, nous avons ajouté ces deux variables affectives aux prédicteurs précédents et nous avons effectué cinq analyses de régression, une pour chacune des motivations à punir (rétribution, compensation, dissuasion individuelle, dissuasion collective et réhabilitation). Enfin, nous avons effectué une analyse de régression pour la punition en ajou- 
Tableau 1 : Notes moyennes aux différentes variables dépendantes selon l'importance des conséquences, le statut mental et le passé judiciaire de l'auteur de l'infraction (notes de 1 à 7 )

\begin{tabular}{|c|c|c|c|c|c|c|c|c|}
\hline & \multicolumn{4}{|c|}{ Conséquences Faibles } & \multicolumn{4}{|c|}{ Conséquences Fortes } \\
\hline & \multicolumn{2}{|c|}{$\begin{array}{c}\text { Pleine Possession } \\
\text { Moyens }\end{array}$} & \multicolumn{2}{|c|}{$\begin{array}{l}\text { Traumatisme } \\
\text { Psychique }\end{array}$} & \multicolumn{2}{|c|}{$\begin{array}{c}\text { Pleine Possession } \\
\text { Moyens }\end{array}$} & \multicolumn{2}{|c|}{$\begin{array}{l}\text { Traumatisme } \\
\text { Psychique }\end{array}$} \\
\hline & $\begin{array}{l}\text { Primo- } \\
\text { Délinq }\end{array}$ & Récidiv. & $\begin{array}{l}\text { Primo- } \\
\text { Délinq }\end{array}$ & Récidiv. & $\begin{array}{l}\text { Primo- } \\
\text { Délinq }\end{array}$ & Récidiv. & $\begin{array}{l}\text { Primo- } \\
\text { Délinq. }\end{array}$ & Récidiv \\
\hline $\begin{array}{l}\text { Gravité } \\
\text { Acte }\end{array}$ & 3.62 & 3.63 & 3.54 & 3.20 & 4.84 & 4.88 & 4.72 & 5.00 \\
\hline $\begin{array}{l}\text { Contrôl. } \\
\text { Auteur }\end{array}$ & 6.45 & 6.51 & 3.46 & 3.64 & 6.36 & 6.40 & 3.20 & 3.50 \\
\hline $\begin{array}{l}\text { Stabilité } \\
\text { Auteur }\end{array}$ & 4.70 & 2.37 & 5.42 & 2.56 & 4.60 & 2.12 & 5.64 & 2.79 \\
\hline $\begin{array}{l}\text { Respons. } \\
\text { Auteur }\end{array}$ & 6.58 & 6.77 & 4.19 & 4.40 & 6.44 & 6.48 & 3.72 & 4.50 \\
\hline $\begin{array}{l}\text { Souffr. } \\
\text { Victime } \\
\text { Proba. }\end{array}$ & 4.95 & 4.55 & 4.07 & 4.60 & 5.60 & 6.08 & 5.88 & 6.00 \\
\hline $\begin{array}{l}\text { Récidiv. } \\
\text { Auteur }\end{array}$ & 4.62 & 5.77 & 4.00 & 6.04 & 4.40 & 5.88 & 4.24 & 6.04 \\
\hline $\begin{array}{l}\text { Colère } \\
\text { Auteur }\end{array}$ & 4.70 & 4.33 & 3.26 & 3.84 & 5.00 & 5.32 & 4.28 & 4.45 \\
\hline $\begin{array}{l}\text { Sympat. } \\
\text { Victime }\end{array}$ & 4.79 & 4.48 & 4.52 & 4.76 & 4.64 & 5.08 & 5.36 & 5.29 \\
\hline $\begin{array}{l}\text { Motiv. } \\
\text { Rétribu. }\end{array}$ & 5.33 & 4.88 & 4.07 & 4.40 & 5.44 & 5.40 & 3.88 & 4.00 \\
\hline $\begin{array}{l}\text { Motiv. } \\
\text { Compen. } \\
\text { Motiv. }\end{array}$ & 3.87 & 3.92 & 3.69 & 3.96 & 3.72 & 4.04 & 4.52 & 4.79 \\
\hline $\begin{array}{l}\text { Dissua. } \\
\text { Individ. } \\
\text { Motiv. }\end{array}$ & 5.87 & 5.29 & 4.53 & 5.12 & 5.48 & 5.44 & 5.20 & 4.62 \\
\hline $\begin{array}{l}\text { Dissua. } \\
\text { Coll. }\end{array}$ & 4.50 & 4.59 & 3.34 & 4.24 & 3.88 & 4.20 & 4.24 & 4.04 \\
\hline $\begin{array}{l}\text { Motiv. } \\
\text { Réhabil. }\end{array}$ & 5.45 & 5.59 & 4.57 & 5.00 & 5.36 & 5.24 & 5.12 & 4.87 \\
\hline $\begin{array}{l}\text { Sévérité } \\
\text { Punition }\end{array}$ & 3.79 & 4.44 & 2.30 & 3.64 & 4.40 & 4.88 & 3.44 & 4.41 \\
\hline $\begin{array}{l}\text { Type de } \\
\text { punition }\end{array}$ & 3.88 & 4.44 & 2.92 & 3.68 & 4.92 & 5.84 & 4.56 & 5.25 \\
\hline
\end{tabular}

tant aux prédicteurs précédents les cinq motivations à punir. Nous avons utilisé comme critère la sévérité de la punition et non pas le type de punition en raison des mauvaises qualités psychométriques de cette dernière mesure (hétérogénéité des punitions proposées) ${ }^{4}$.
Les résultats de l'analyse des pistes causales vont dans le sens de nos hypothèses. Ainsi, conformément à l'hypothèse 1 , la contrôlabilité est associée positivement à la responsabilité de l'auteur $(F(1,398)$ $=911.22 ; \beta=+0.83 ; p<.001)$ : plus l'estimation de la contrôlabilité est élevée et plus la responsabilité de l'auteur tend à être jugée forte (voir figure 
1). La responsabilité détermine ensuite pour partie (lien significatif positif) la colère éprouvée à l'égard de l'auteur de l'acte $(F(1,393)=40.96 ; \beta=+0.33$; $p<.001)$. Ensuite, la colère est significativement prédictive (lien positif) de la motivation rétributive $(F(1,391)=44.28 ; \beta=+0.33 ; p<.001)$, la motivation rétributive influençant pour partie (lien significatif positif) la sévérité de la punition $(F(1,386)=$ $21.01 ; \beta=+0.23 ; p<.005)$. De même, en accord avec l'hypothèse 2 , la gravité détermine pour partie (lien significatif positif) le degré de souffrance de la victime $(F(1,398)=212.32 ; \beta=+0.59 ; p<.001)$ qui à son tour tend à influencer (lien significatif positif) la sympathie à l'égard de la victime $(F(1,393)=$ $51.78 ; \beta=+0.41 ; p<.001)$. Ensuite, on note bien que la sympathie est pour partie prédictive (lien significatif positif) de la motivation compensative $(F(1$, $391)=13.61 ; \beta=+0.19 ; p<.001)$, cette dernière étant associée pour partie (lien significatif positif) à la sévérité de la punition $(F(1,386)=14.02 ; \beta=$ $+0.17 ; p<.002$ ). Par contre, les hypothèses 3 , 4 et 5 qui portaient sur les motivations utilitaristes (dissuasion individuelle, collective et réhabilitation) ne sont que partiellement validées. Ainsi, si effectivement la stabilité est prédictive pour partie (lien significatif positif) de la probabilité de récidive $(F(1,398)=$ $83.57 ; \beta=+0.42 ; p<.001)$, on ne constate pas de liens de dépendance significatifs, ni entre la probabilité de récidive et les trois motivations utilitaristes, ni entre la dissuasion collective et la sévérité de la punition. Toutefois, on note bien que la dissuasion individuelle est reliée pour partie (lien significatif positif) à la sévérité de la punition $(F(1,386)=5.94$; $\beta=+0.10 ; p<.01)$ et que la motivation réhabilitative est reliée pour partie (lien significatif négatif) à la sévérité de la punition $(F(1,386)=10.70 ; \beta=$ $-0.13 ; \mathrm{p}<.001)$. Dans ce cas, cela signifie que plus la justification de la punition par la motivation réhabilitative est élevée et moins la sévérité de la punition tend à être importante. Enfin, conformément à l'hypothèse 6 , on note bien un lien direct (significatif et positif) entre la gravité et la sévérité de la punition $(F(1,386)=41.64 ; \beta=+0.29 ; p<.001)$.

Par ailleurs, on note des relations non prévues. Ainsi, on remarque d'abord un effet direct de la gravité sur la colère à l'égard de l'auteur : plus la gravité est importante et plus la colère à l'égard de l'auteur tend à être élevée $(F(1,398)=15.24 ; \beta=+0.30 ; p<.001)$. Ensuite on note, d'une part que la colère à l'égard de l'auteur est prédictive pour partie (liens significatifs positifs) de la dissuasion individuelle $(F(1,391)=$ $33.03 ; \beta=+0.31 ; p<.001)$ et de la dissuasion collective $(F(1,391)=24.68 ; \beta=+0.29 ; p<.001)$; d'autre part, que la responsabilité de l'auteur influence négativement la motivation réhabilitative $(F(1,391)=$ $15.89 ; \beta=-0.23 ; p<.001)$ : plus la responsabilité est forte et plus la motivation réhabilitative tend à être faible. Enfin, on relève que la probabilité de la récidive s'associe pour partie (lien significatif positif) à la sévérité de la punition $(F(1,386)=21.27 ; \beta=$ $+0.17 ; p<.001)$. Remarquons pour terminer que la part de variance expliquée $\left(R^{2}\right)$ est assez importante pour la souffrance de la victime $(36 \%)$, la colère à l'égard de l'auteur $(32 \%)$ et la motivation rétributive $(27 \%)$, importante pour la sévérité de la punition $(57 \%)$, voire très importante en ce qui concerne la responsabilité de l'auteur $(70 \%)$.

\section{Discussion}

L'objectif de cette recherche était de préciser les relations entre différents déterminants de la punition, la punition étant envisagée ici, en référence aux travaux de Weiner, comme le résultat d'un processus par étapes. Pour cela, nous avons mis en perspective deux champs d'étude connexes, l'un relatif aux travaux sur l'attribution de responsabilité et de punition, l'autre relatif aux travaux sur les motivations à punir. Dans ce cadre, nous avons pris en compte la gravité de l'infraction, les caractéristiques de l'auteur de l'infraction et des facteurs affectifs associés à l'auteur ou à la victime. Nous nous sommes également intéressés aux motivations à punir en distinguant les motivations dissuasives individuelle et collective et en intégrant dans l'étude les motivations réhabilitative et compensative.

Si on réfère dans un premier temps aux analyses préliminaires, on note que les résultats sont cohérents avec le sens commun. Ainsi, la responsabilité de l'auteur est jugée plus forte quand il a agi en pleine possession de ses moyens ou qu'il est récidiviste. De même, la colère à l'encontre de l'auteur et la sympathie à l'égard de la victime sont plus élevées quand les conséquences sont fortes. De même encore, la sévérité de la punition et le type de punition sont plus importants quand l'auteur a agi en pleine possession de ses moyens et qu'il est récidiviste mais aussi quand les conséquences sont fortes. En accord avec la logique rétributive qui met l'accent sur le libre-arbitre de l'auteur, on remarque que le recours à la motivation rétributive est plus élevé quand l'auteur a agi en pleine possession de ses moyens. Enfin, en accord avec la logique compensative qui met en avant la souffrance de la victime, on note que le recours à cette motivation est plus important quand les conséquences pour la victime sont fortes. 
Figure 1 :Diagramme de l'analyse des pistes causales

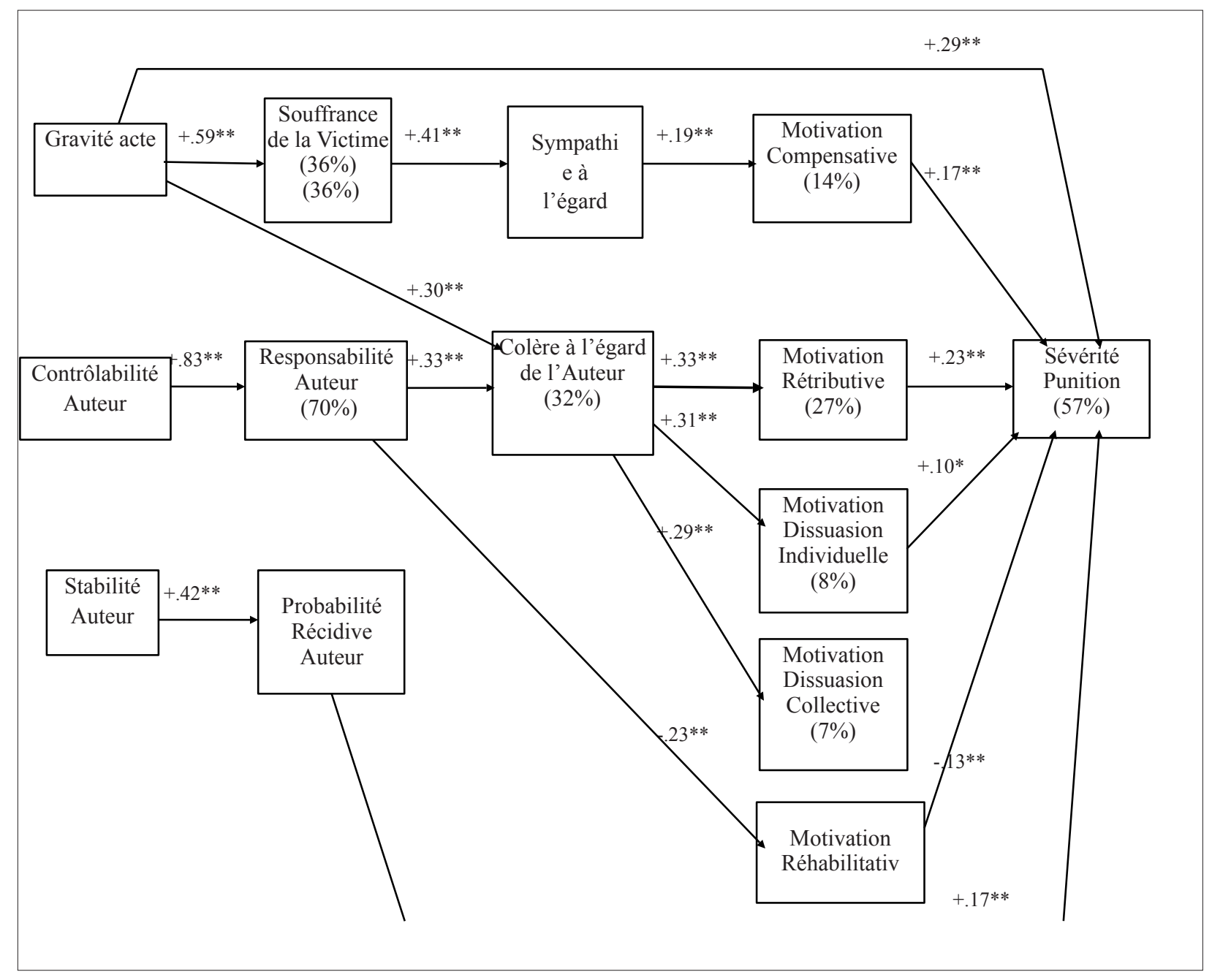

Note : ne sont indiqués que les liens significatifs (coefficients standardisés betas) : $*<<.01 . * * p<.001$. Entre parenthèses : $R^{2}$ (pourcentage de variance expliquée).

En ce qui concerne l'analyse des pistes causales, les résultats vont dans le sens d'un processus en étapes, la plupart des liens étant successifs. Plus précisément, en accord avec l'hypothèse 1 , on constate bien des liens positifs successifs entre la contrôlabilité, la responsabilité de l'auteur, la colère à l'égard de l'auteur, la motivation rétributive et la sévérité de la punition. Ce résultat est similaire à celui déjà constaté par Weiner (Weiner et al., 1997). Les résultats confortent également l'hypothèse 2 (liens successifs positifs entre gravité de l'infraction, souffrance de la victime, sympathie à l'égard de la victime, motivation compensative, punition). Par ailleurs, on remarque que c'est la motivation rétributive qui contribue le plus à la punition (si on se réfère à la part de variance expliquée), ce qui peut s'expliquer par le fait que dans notre droit pénal, cette justification de la punition est centrale (Carbasse, 2000). La seconde contribution en importance à la punition provient de la motivation compensative. Cela montre l'intérêt de prendre en compte cette motivation qui est pour l'instant rarement mesurée dans les recherches expérimentales. La contribution significative de cette motivation est cohérente avec les analyses sociologiques actuelles qui soulignent le développement d'une logique pénale centrée sur les victimes (Rauschenbach et Languin, 2007). Par contre, les résultats ne valident pas les hypothèses relatives aux motivations utilitaristes (hypothèses 3, 4 et 5 ). $\mathrm{Si}$, comme on pouvait le supposer, la stabilité du comportement 
infractionnel de l'auteur influence effectivement l'estimation de la probabilité de récidive, on ne remarque cependant pas d'influence de la probabilité de récidive sur les différentes motivations utilitaristes. Ici peut-être aurait-il fallu, pour mettre en évidence de tels liens, aller au-delà du simple contraste entre primo-délinquant et récidiviste en prenant en compte dans nos vignettes des niveaux de récidives plus ou moins importants. Toutefois, on remarque comme attendu, un lien entre certaines motivations utilitaristes et la punition. Ainsi, la dissuasion individuelle est bien reliée positivement à la sévérité de la punition. De même, la motivation réhabilitative est bien reliée négativement à la sévérité de la punition. Par contre, il n'y a pas de lien entre la dissuasion collective et la punition. L'effet d'un côté de la dissuasion individuelle sur la punition, l'absence d'effet de l'autre côté de la dissuasion collective sur la punition, conforte l'intérêt de distinguer ces deux motivations. Toutefois, cette absence d'effet de la dissuasion collective sur la punition est peut-être liée au format de notre expérience qui n'était pas nécessairement adapté. En effet, notre expérience décrivant à chaque fois un fait divers précis, portant sur un cas individuel (l'auteur de l'infraction), les sujets ont peut-être considérés que la question de la dissuasion dans sa dimension collective n'était pas pertinente. Par ailleurs, à propos de la probabilité de récidive, si celle-ci n'agit pas, comme on l'a indiqué, sur les motivations utilitaristes, remarquons cependant qu'elle est reliée positivement à la punition. Ce résultat est cohérent avec le régime de la peine, le code pénal (Wackenheim, 2003), qui prévoit des punitions plus fortes pour les récidivistes. D'autre part, on note que le recours aux motivations utilitaristes est influencé par certains facteurs. Ainsi, à propos de la motivation réhabilitative, on constate que moins la responsabilité de l'auteur est élevée et plus les sujets tendent à mettre l'accent sur cette motivation. Cela présente une certaine cohérence avec la philosophie de la motivation réhabilitative qui met en avant, pour légitimer le fait de donner une nouvelle chance aux individus fautifs, le poids du milieu dans la genèse de la délinquance (Garapon et al., 2001). Autrement dit, cette philosophie en insistant sur les déterminismes sociaux, tend à minorer la responsabilité individuelle des infractions. On remarque également que les motivations dissuasives individuelles et collectives sont influencées par la colère éprouvée à l'égard du délinquant. Cela semble suggérer l'impact de facteurs affectifs sur ces motivations. Ici, il est tentant de relier ce phénomène aux évolutions pénales contemporaines.
Actuellement, dans de nombreux pays occidentaux (et spécialement aux États-Unis), l'optique dissuasive se développe au travers de discours qui mettent l'accent sur la nécessité de peines dissuasives plus longues. Or ces discours sont alimentés, pour partie, par la survenue de faits divers qui suscitent des réactions émotives fortes, notamment de colère, de la part de l'opinion publique (Salas, 2005). Par ailleurs, les résultats vont dans le sens de l'hypothèse 6 puisque l'on constate bien un lien direct entre la gravité et la sévérité de la punition. Ce résultat est cohérent avec les travaux sur le biais lié aux conséquences (Robbennolt, 2000). Pour conclure sur nos différents résultats, on note que ce sont la gravité et la colère qui ont le plus d'impact. Ces deux dimensions agissent en effet sur plusieurs variables dépendantes à la fois. Ainsi, la gravité agit à la fois sur la souffrance de la victime, la colère à l'égard de l'auteur et la punition tandis que la colère à l'égard de l'auteur influence la motivation rétributive et les deux motivations dissuasives (individuelle et collective).

Précisons pour terminer qu'il convient d'être prudent dans la généralisation de ces résultats. D’abord, les résultats de l'analyse des pistes causales, comme dans le cas de toute méthode d'équations structurelles, sont sensibles à la façon dont sont construites les droites de régression (Grimm et Yarnold, 1995). Ensuite, on peut supposer que les processus d'attribution de punitions soient influencés, d'une part par la nature des délits et des crimes présentés (Miller, Rossi et Simpson, 1991), d'autre part par certaines variables traditionnellement associées aux représentations de la délinquance (sentiment d'insécurité, niveau d'intégration sociale, etc.) (Amerio et Roccato, 2004). Enfin, comme l'indique certains travaux, des dimensions de nature normative (phénomènes de stigmatisation sociale de l'auteur de l'infraction, statut social de l'auteur) (Hamilton et Rytina, 1980 ; Oberlé et Gosling, 2004) ou motivationnelle (attribution défensive, croyance en un monde juste) (Alicke, 2000) peuvent également modifier ces processus d'attribution de punition. Cela dit, nous espérons que cette recherche montre l'intérêt, afin de mieux comprendre les déterminants de la punition, de prendre en compte à la fois les facteurs cognitifs et affectifs et d'y associer les motivations à punir des individus. Et sur ce dernier point, de l'utilité d'étudier la motivation compensative qui pour l'instant, du moins à notre connaissance, ne fait pas encore l'objet d'études expérimentales précises. 


\section{NOTES}

1. Nous avons décidé de faire varier l'importance des conséquences en manipulant le degré d'atteinte physique de la victime. Nous avons fait ce choix car c'est un élément cité prioritairement par les personnes dans les enquêtes de victimation pour juger de la gravité d'un acte. Néanmoins, nous sommes conscients du fait que cela peut introduire une difficulté méthodologique, les deux niveaux de conséquences pouvant faire aussi varier la nature de l'acte : dans le premier cas il s'agit d'un vol accompagné de violences (vol à l'arraché), dans le second cas d'un vol et d'une violence. De plus, cette manipulation comporte le risque d'interférer avec la variable statut mental, les sujets pouvant se demander (en situation conséquences fortes) si le fait de violemment frapper la victime n'est pas le signe d'une certaine instabilité mentale. Il semble que cela ne soit cependant pas le cas. En effet, une analyse de variance montre que l'estimation du statut mental de l'auteur est similaire en conséquences faibles et fortes $(F<1)$ (estimation qui était appréhendée par la question sur le degré de contrôle de l'auteur de l'infraction. $c f$. infra variables dépendantes).

2. Lors d'un pré-test, on a présenté à 30 étudiantes et 30 étudiants des textes décrivant les différentes motivations à punir, puis on proposait différents items appartenant à l'une ou l'autre de ces motivations, et les sujets devaient les classer selon ces cinq motivations. On a retenu pour la présente étude, les items qui avaient été correctement classés par tous les sujets dans la motivation à punir correspondante.

3. Rappelons que la note 3 correspondait à trois mois de prison avec sursis, la note 4 à trois mois de prison ferme, la note 5 à six mois de prison ferme.

4. La sévérité de la punition et le type de punition sont cependant fortement corrélés $(R$ de Bravais-Pearson $=+0.78 ; p<.001)$

\section{RÉFÉRENCES}

- Alicke, M.D. (1990). Incapacitating conditions and alteration of blame. Fournal of Social Behavior and Personality, 6, pp. 651-664.

- Alicke, M.D. (2000). Culpable control and the psychology of blame. Psychological Bulletin, 126, pp. 556-574.

- Amerio, P. et Roccato, M. (2004). Structure multifacettée du sentiment d'insécurité. Psychologie et Société, 7, pp. 47-70.

- Barillon, J. et Bensussan, P. (2004). Le désir criminel. Paris, Editions Odile Jacob.

- Beccaria, C. (1991). Traité des délits et des peines. Paris, Flammarion. (1 ${ }^{\text {ère }}$ publication 1764).

- Bentham, J. (1802). Traité de législation civile et pénale. Paris, Bossange et Besson. (1 ${ }^{\text {ere }}$ publication 1789).

- Carbasse, J.M. (2000). Histoire du droit pénal et de la justice criminelle. Paris, Presses Universitaires de France.

- Garlsmith, K.M, Darley, J.M. et Robinson, P.H. (2002). Why do we punish? Deterrence and just deserts as motives for punishment. Fournal of Personality and Social Psychology, 83, pp. 284-299.

- Cesoni, M.L. et Rechtman, R. (2005). La réparation psychologique de la victime : une nouvelle fonction de la peine ? Revue de Droit Pénal et de Criminologie, 2, pp. 158-178.

- Darley, J.M., Carlsmith, K.M. et Robinson, P.H. (2000). Incapacitation and just deserts as motives for punishment. Law and Human Behavior, 24, pp. 659-683.

- De Keijser, J.W., Van der Leeden, R. et Jackson, J.L. (2002). From moral theory to penal attitudes and back: A theoretically integrated modeling approach. Behavioral Sciences and the Law, 20, pp. 317-335.

- Fauconnet, p. (1920). La responsabilité. Paris, Felix Alcan.

- Fincham, F.D. et Jaspars, J.M. (1980). Attribution of responsibility: From man the scientist to man as lawyer. In L. Berkowitz (Dir.), Advances in Experimental Social Psychology. New-York, Academic Press, vol.13, pp. 81-138
- Finkel, N.J., Maloney, S.T., Valbuena, M.Z. et Groscup, J. (1996). Recidivism, proportionalism, and individualized punishment. American Behavioral Scientist, 39, pp. 474-487.

- Fischer, G.N. (2003). Psychologie des violences sociales. Paris, Dunod.

- Garapon, A., Gros, F. et Pech, T. (2001). Et ce sera justice : punir en démocratie. Paris, Editions Odile Jacob.

- Garapon, A. et Salas, D. (1996). La république pénalisée. Paris, Hachette.

- Golberg, J.H., Lerner, J.S. et Tetlock, P.E. (1999). Rage and reason: The psychology of the intuitive prosecutors. European Fournal of Social Psychology, 29, 5-6, pp. 781-795.

- Graham, S., Weiner, B. et Zucker, G.S. (1997). An attributional analysis of punishment goals and public reactions to O.J. Simpson. Personality and Social Psychology Bulletin, 23, pp. 331-346.

- Grimm, L.G. et Yarnold, P.R. (1995). Reading and understanding multivariate statistics. Washington, American Psychological Association.

- Haidt, J., Koller, S.H. et Dias, M.G. (1993). Affect, culture and morality, or is it wrong to eat your dog ? Fournal of Personality and Social Psychology, 65, 4, pp. 613-628.

- Hamilton, V.L. et RYtina, s. (1980). Social consensus on norms of justice: Should the punishment fit the crime? American Fournal of Sociology, 85, 5, pp. 1117-1144.

- Hamilton, V.L. et Sanders, J. (1981). The effect of roles and deeds on responsability judgments: The normative structure of wrongdoing. Social Psychology Quarterly, 4, pp. 237-254.

- Hegel, F. (1982). Principes de la philosophie du droit. Paris, Vrin. (1 $1^{\text {ère }}$ publication 1821).

- Heider, F. (1944). Social perception and phenomenal causality. Psychological Reviere, 51, pp. 358-374.

- Heider, F. (1958). The psychology of interpersonal relations. New-York, Wiley.

- Hogarth, J. (1971). Sentencing as a human process. Canada, Toronto, University of Toronto Press.

- Kant, E. (1971). Doctrine du droit. Paris, Vrin. (1 ${ }^{\text {ère }}$ publication 1796). - Lagrange, H. (2003). Demandes de sécurité. Paris, Le Seuil.

- Larguier, J. (2001). Droit pénal général. Paris, Dalloz.

- Lerner, J.S., Golberg, J.H. et Tetlock, P.E. (1998). Sober second thought: The effects of accountability, anger, and authoritarianism on attributions of responsibility. Personality and Social Psychology Bulletin, 24, 6, pp. 563-574.

- Lerner, M.J. (1998). The two forms of belief in a just world. In L. Montada et M.J. Lerner (Dirs.), Responses to victimizations and belief in a just word. New-York, Plenum Press, pp. 247-269.

- Mazzocco, P.J., Alicke, M.D. et Davis, T.L. (2004). On the robustness of outcome bias: No constraint by prior culpability. Basic \& Applied Social Psychology, 26, 2/3, pp. 131-146.

- McFatter, R.M. (1978). Sentencing strategies and justice: Effects of punishment philosophy on sentencing decisions. Fournal of Personality and Social Psychology, 36, pp. 1490-1500.

- Miller, J.L., Rossi, P.H. et Simpson, J.E. (1991). Felony punishments: A factorial survey of perceived justice and criminal sentencing. The Fournal of Criminal Law and Criminology, 82, pp. 396-415.

- Oberlé, D. et Gosling, P. (2004). Intention, responsabilité, sanction : l'intention est-elle un préalable ou une justification de la sanction ? In J.L. Beauvois, R.V. Joulé et J.M. Monteil (Dirs.), Perspectives Cognitives et Conduites Sociales. Rennes, Presses Universitaires de Rennes, vol. 9, pp. 271-291.

- Oswald, M.E., Hupfeld, J., Klug et Gabriel, U. (2002). Layperspectives on criminal deviance, goals of punishment, and punitivity. Social fustice Research, 15, pp. 85-98.

- Oswald, M.E., Orth, U., Aeberhard, M. et Schneider, E. (2005). Punitive reaction to completed crimes versus accidentally uncompleted crimes. Fournal of Applied Social Psychology, 35, 4, pp. 718-731. 
- Piaget, J. (1932). Le jugement moral chez l'enfant. Paris, Félix Alcan.

- Rauschenbach, M. et Languin, N. (2007) Law as a product of emotion: The rise of the victim. Université de Genève. Tapuscrit non publié.

- Robbennolt, J.K. (2000). Outcome severity and judgments of "responsibility": A meta-analytic review. Fournal of Applied Social Psychology, 30, pp. 2575-2609.

- Rucker, D.D., Polifroni, M., Tetlock, P.E. et Scott, A.L. (2004). On the assignment of punishment: The impact of general-societal threat and the moderating role of severity. Personality and Social Psychology Bulletin, 30, pp. 673-684.

- Salas, D. (2005). La volonté de punir. Essai sur le populisme pénal. Paris, Hachette.

- Saleilles, R. (1898). L'individualisation de la peine. Paris, Félix Alcan.

- SHAVER, K.G. (1985). The attribution of blame: Causality, responsibility, and blameworthiness. New-York, Springer-Verlag.

- Shultz, T.R., Schleifer, M. et Altman, I. (1981). Judgments of causation, responsibility, and punishment in cases of harm-doing. Canadian Fournal of Behavioural Science, 13, pp. 238-253.

- Steffensmeier, D. et Kramer, J.H. (1982). Sex-based differences in the sentencing of adult criminal defendants: An empirical test and theoretical overview. Sociology and Social Research, 3, pp. 289-304.

- Tabachnick, B.G. et Fidell, L.S. (2001). Using multivariate statistics. Boston, Allyn \& Bacon.

- Tetlock, P.E. (2002). Social functionalist frameworks for judgment and choice: Intuitive politicians, theologians, and prosecutors. Psychological Reviere, 109, pp. 451-471.
- Tocoueville, A. (1984). Ecrits pénitentiaires. Paris, Gallimard. (1 ${ }^{\text {ère }}$ publication 1833).

- Tyler, T. et Boeckmann, R. (1997). Three strikes and you are out, but why? The psychology of public support for punishing rule breakers. Law and Society Review, 31, pp. 237-264.

- Van de Kerchove, M. (2005). Les fonctions de la sanction pénale. Informations Sociales, 127, pp. 22-31.

- Vidmar, N. et Miller, D. (1980). Social psychological processes underlying attitudes toward legal punishment. Law and Society Review, 14 , pp. 565-602.

- Viney, G. (1990). La responsabilité. Archives de Philosophie du Droit, 35, pp. 175-292.

- Wackenheim, V. (2003). Le code pénal. Paris, Editions Prat.

- WeINER, B. (1995). Judgments of responsibility: A foundation for a theory of social conduct. New-York, Guilford Press.

- Weiner, B. (2001). Responsibility for social transgressions: An attributional analysis. In B.F. Malle (Dir.), Intentions and intentionality: Foundations of social cognition. Cambridge, MIT Press, pp. 331-344.

- Weiner, B., Graham, S. et Reyna, C. (1997). An attributional examination of retributive versus utilitarian philosophies of punishment. Social fustice Research, 10, pp. 431-451. 